\title{
Interparticle gap distributions on one-dimensional lattices
}

\author{
Maria R D'Orsogna ${ }^{1,3}$ and Tom Chou $^{2}$ \\ ${ }^{1}$ Department of Chemistry and Chemical Engineering, California Institute of Technology, \\ MC 210-41 Pasadena, CA 91125, USA \\ 2 Department of Biomathematics, UCLA, Los Angeles, CA 90095-1766, USA
}

Received 21 September 2004, in final form 16 November 2004

Published 23 December 2004

Online at stacks.iop.org/JPhysA/38/531

\begin{abstract}
We analyse the successive binding of two species of particles on a onedimensional discrete lattice, where the second variety is deposited only after complete adsorption of the first. We consider the two extreme cases of a perfectly irreversible initial deposition, with non-sliding particles, and that of a fully equilibrated one. For the latter we construct the exact gap distribution from the Tonks gas partition function. This distribution is contrasted with that obtained from the random sequential adsorption process. We discuss implications for the kinetics of adsorption of the second species, as well as experimental relevance of our results.
\end{abstract}

PACS numbers: 05.70.Ln, 68.43.De, 02.50.-r

(Some figures in this article are in colour only in the electronic version)

\section{Introduction}

Particle deposition onto a substrate is one of the most frequent phenomena encountered in chemical, condensed matter and biophysical systems. Proteins, cells and colloids adsorb on solid surfaces, and depending on the specific nature of the particle-particle and particlesubstrate interactions, different kinetic descriptions of adsorption are possible [1-3]. Because of the complexity of the problem, analytical treatments are available only for one-dimensional models and one generally has to resort to approximations or computational methods for higher dimensions [4]. This should not be seen merely as a limitation: many microscopic processes, such as adsorption on DNA, can be approximated as linear and often the one-dimensional results offer insight and checks for numerical studies in higher dimensions.

One of the most studied models to describe particle adsorption is the random sequential adsorption (RSA) process, also known as the car-parking model [5-7]. The essential feature of RSA is its irreversibility: once a particle of length $k$ has been randomly deposited along the linear chain it cannot detach or further diffuse. The random deposition process generates

\footnotetext{
3 Present address: Department of Mathematics, UCLA, Los Angeles, CA 90095.
} 
gaps of empty sites, and no adsorbate overlaps are allowed: each subsequent particle of length $k$ must be able to accommodate itself in a vacant space large enough for deposition. At saturation, the chain is left with gaps of length $m<k$ which cannot be further filled in, and its average coverage is $\theta_{\infty}(k)<1$.

The RSA model has proven to be quite successful in describing a number of systems [8], lattice and continuum limits have been studied $[9,10]$ and features such as particle cooperativity [10], size mixing and competitiveness [11] have been taken into account. Many other variants of RSA have been considered [1-3], among which a continuum model of adsorption where irreversibility is relaxed and particle desorption and sliding during the deposition process are allowed [12]. In this context, an important question is to what extent the post-adsorption sliding of particles on the chain can be neglected. Some experimental measurements of twodimensional protein adsorption $[13,14]$ for instance, suggest that there might be a diffusional equilibration of adsorbates at play. Particle sliding after random deposition has also been presented as a mechanism for certain proteins to find their target site on DNA segments [15], as opposed to three-dimensional spatial diffusion in solution. The observational time scales involved might also influence the correct picture for adsorbate mobility. For particles of diffusivity $D$ and adsorption rate $r$ the kinetics of the system can be considered irreversible if the diffusional time scales are much larger than those associated with deposition, $k^{2} / D \ll 1 / r$. On the other hand, if one waits long enough and assumes that after the initial adsorption no other particle hits the substrate $(r \rightarrow 0)$, a diffusional equilibration occurs, and the particles would be then described by a one-dimensional Tonks gas [16].

While it is experimentally feasible to determine the jamming coverage for most systems of interest, this is not enough to distinguish the irreversible from the equilibrated deposition processes, since the two might exhibit the same coverage. The pair correlation function has been proposed as a discriminant $[17,18]$, with the equilibrated process exhibiting large oscillations absent in irreversible, saturated RSA. Another consideration worth exploring is that post-deposition particle sliding influences the distribution of gaps of empty sites. If we consider the subsequent adsorption of a second species onto such gaps, its statistics will necessarily depend on the mobility of the first species. We may then use a secondary deposition process as a probe of the initial gap statistics.

The aim of this paper is to study the gap distributions of one-dimensional particles that exclude each other in the two extreme limits of irreversible RSA (both infinite- and finitesized systems), and in that of a fully equilibrated ensemble of particles, a Tonks gas. We provide expression for computing the two limiting gap distributions and contrast them within the context of a second adsorbate, possibly of a different size, irreversibly binding on the substrate. In both limits, formulae are derived for the gap distributions as a function of time, particle lengths and binding cooperativity. These results are potentially valuable for understanding the physics behind more complex competitive binding phenomena, such as the order-of-addition effect of SSB/RecA $[19,20]$. Possible realizations of competitive binding and experimental measurements are proposed.

\section{Gap distributions in RSA}

In most lattice descriptions, the one-dimensional RSA process is analysed on an infinite chain. The probability $\mathcal{P}(m, k, t)$ that after deposition of $k$-mers for a time $t$, any randomly picked $m$ contiguous sites of the infinite lattice belong to a gap of length $m$ or more is [6]

$\dot{\mathcal{P}}(m, k, t)=-(k-m+1) \mathcal{P}(k, k, t)-2 \sum_{j=1}^{m-1} \mathcal{P}(k+j, k, t) \quad$ for $\quad m<k$ 
and

$\dot{\mathcal{P}}(m, k, t)=-(m-k+1) \mathcal{P}(m, k, t)-2 \sum_{j=1}^{k-1} \mathcal{P}(m+j, k, t), \quad$ for $\quad m \geqslant k$,

where we have set the deposition rate $r=1$. One of the earliest derivations of these expressions, for $k=2$, and their physical significance is explained in detail in [6]. Equation (2) for instance arises from the observation that there are $(m-k+1)$ ways to accommodate a $k$-mer into a gap of length $m$, thus decreasing $\mathcal{P}(m, k, t)$. This is the origin of the first term on the right-hand side. A gap of length $m$ or more can be destroyed also by placing the $k$-mer on the edges of a selected gap of length $m$. There are $k-1$ possibilities for this to happen, each corresponding to $j=1,2, \ldots, k-1$ sites of the $k$-mer outside of the selected gap of length $m$. In this case, however, the annihilating gap must be at least of length $m+j$ because of the nonoverlapping condition. This process can occur on the left or on the right edge of the selected gap of length $m$, hence the factor 2 in the second term of the rhs of equation (2). Similar considerations apply to the derivation of equation (1). The solution to equations (1) and (2) is well known:

$\mathcal{P}(m, k, t)=1-\int_{0}^{t} \mathrm{~d} u\left[(k-m+1)+2 \sum_{j=1}^{m-1} \mathrm{e}^{-j u}\right] \mathrm{e}^{-u} F(k, u) \quad$ for $\quad m<k$

$\mathcal{P}(m, k, t)=\mathrm{e}^{-(m-k+1) t} F(t, k) \quad$ for $\quad m \geqslant k$

where

$$
F(k, t)=\exp \left[-2 \sum_{j=1}^{k-1} \frac{1-\mathrm{e}^{-j t}}{j}\right]
$$

is independent of $m$. The coverage of the system $\theta(k, t)$ is given by $\theta(k, t)=1-\mathcal{P}(1, k, t)$. For dimers impinging on finite-size systems of length $M$, it is possible to modify equations (1) and (2) and find the corresponding expressions under periodic and open boundary conditions [25]. In this context, the analytical treatment for $k>2$ is limited to asymptotic expressions for short and long times. Alternatively, exact solutions for general $k$ can be found if one works directly with the total number of gaps of size $m$, which we denote by $\mathcal{N}(m, k, t)$. Following the method outlined by Boucher [23, 24],

$\dot{\mathcal{N}}(M, k, t)=-(M-k+1) \mathcal{N}(M, k, t)$,

$\dot{\mathcal{N}}(m, k, t)=2 \sum_{j=k+m}^{M} \mathcal{N}(j, k, t)-(m-k+1) \mathcal{N}(m, k, t) \quad$ for $\quad k \leqslant m<M$,

$\dot{\mathcal{N}}(k-p, k, t)=2 \sum_{j=2 k-p}^{M} \mathcal{N}(j, k, t) \quad$ for $\quad 1 \leqslant p<k$.

In the above expressions $\mathcal{N}(m, k, t)$ will decrease by the deposition of a $k$-mer onto a gap of length $m$; there are $(m-k+1)$ ways for this to happen. At the same time, the deposition of a $k$-mer on a gap of length $j$ will contribute to the creation of a gap of length $m$ in two possible ways, to its left or to its right. Both these effects contribute to equation (7). In addition, equations (6) and (8) take into account, respectively, that gaps of length $M$ cannot be created, and gaps of length $m \leqslant k-1$ cannot be destroyed. The initial condition is $\mathcal{N}(m, k, t=0)=\delta_{m, M}$. Once $k$ and $M$ are given, the analytical expressions for $\mathcal{N}(m, k, t)$ 
can be found by diagonalizing the corresponding dynamical matrix. For finite-sized RSA systems of length $M$ the time-dependent coverage can be written in terms of the $j$-gap density $\rho_{M}(j, k, t)=\mathcal{N}(j, k, t) / M$ as

$$
\theta_{M}(k, t)=1-\sum_{j=1}^{M} j \rho_{M}(j, k, t) .
$$

The asymptotic behavior $\mathcal{N}(j, k, t) \sim \mathrm{e}^{-t}$ occurs if $k=j$ or if $j \geqslant 2 k$. This behavior arises from the fact that gaps of length $k$ will decay with a unitary time constant. In order to originate gaps of length $k$ two possibilities arise: either $j=k$ or such a gap can be generated by deposition. The minimum length required to generate a new gap of length $k$ is $k+k$ : the first $k$-mer is deposited along the $2 k$ segment so that a $k$ gap is created. The finite-size probability $\mathcal{P}_{M}(m, k, t)$ of finding gaps of length $m$ or larger can be constructed from the $\mathcal{N}(m, k, t)$ functions by noting that each gap of exactly length $j$ can accommodate $(j-m+1)$ gaps of length $m$. Hence,

$$
\mathcal{P}_{M}(m, k, t)=\sum_{j=m}^{M}(j-k+1) \frac{\mathcal{N}(j, k, t)}{M}=\sum_{j=m}^{M}(j-k+1) \rho_{M}(j, k, t) .
$$

In the limit of $M \rightarrow \infty$, the finite-size solutions converge to those obtained from the infinite RSA lattice and $\lim _{M \rightarrow \infty} \mathcal{P}_{M}(m, k, t)=\mathcal{P}(m, k, t)$. At saturation, when only gaps of length $j \leqslant k-1$ exist, the summation in equation (10) must be arrested at $j=k-1$. Upon inversion of equation (10) we obtain the exact relationship

$$
\begin{aligned}
\rho_{M}(m, k, t)= & \mathcal{P}_{M}(m, k, t)-2 \mathcal{P}_{M}(m+1, k, t)\left(1-\delta_{k, m}\right) \\
& +\mathcal{P}_{M}(m+2, k, t)\left(1-\delta_{k, m}\right)\left(1-\delta_{k, m+1}\right) .
\end{aligned}
$$

This relationship also holds in the limit $M \rightarrow \infty$ and can be applied directly to equations (3) and (4) to find the infinite length gap density function $\rho_{\mathrm{RSA}}(m, k, t)$ :

$$
\begin{aligned}
\rho_{\mathrm{RSA}}(m, k, t) & =\mathcal{P}(m, k, t)-2 \mathcal{P}(m+1, k, t)\left(1-\delta_{k, m}\right) \\
& +\mathcal{P}(m+2, k, t)\left(1-\delta_{k, m}\right)\left(1-\delta_{k, m+1}\right) .
\end{aligned}
$$

The gap density function, expressed in terms of $\mathcal{P}(m, k, t)$ is defined as the number of gaps of length $m$ per unit length. One can also evaluate the gap distribution $P_{M}(m, k, t)$, defined as the fraction of all the gaps that are length $m$. For finite systems of length $M, \rho_{M}(m, k, t)$ and $P_{M}(m, k, t)$ are related by

$$
P_{M}(m, k, t)=\frac{M}{N_{g}(M, t)} \rho_{M}(m, k, t),
$$

where $N_{g}(M, t)=\sum_{m=0}^{M} \mathcal{N}(m, k, t)=N_{p}(M, t)+1$ is the total number of gaps, and $N_{p}(M, t)$ is the total number of adsorbates. The number of particles, expressed in terms of coverage is $N_{p}(M, t)=M \theta_{M}(k, t) k^{-1}$. In the limit of large $M, N_{g}(M, t) \simeq N_{p}(M, t)$ if $M \theta_{M}(k, t) \gg k$. This condition is violated only at extremely short times, $t \simeq r^{-1}$. Hence,

$$
P_{\mathrm{RSA}}(m, k, t)=k \frac{\rho_{\mathrm{RSA}}(m, k, t)}{\theta(m, k, t)} .
$$

In the following sections, we shall be working with the probability distribution $P_{\mathrm{RSA}}(m, k, t)$ and gap density $\rho_{\mathrm{RSA}}(m, k, t)$ and compare the saturated-RSA values with those obtained from the equilibrated deposition case. 


\section{Gap distributions in cooperative RSA}

In this section we extend equations (1) and (2) to include cooperative effects on an infinite lattice. The rate of adsorption is $r_{1}$ when particles are deposited without touching others and $r_{2}$ when they stick to their neighbours. The time evolution of $\mathcal{P}\left(m, k, r_{1}, r_{2}, t\right) \equiv \mathcal{R}(m)$ is obtained through equation (10), which is valid also in the limit $M \rightarrow \infty$, and by evaluating the possible ways to accommodate a $k$-mer in a gap of exact length $m$ with or without touching other neighbours. This is equivalent to modifying equations (6)-(8) by taking into account the existence of two different rates and then constructing $\mathcal{P}_{M}(m, k, t)$ with $M \rightarrow \infty$. We obtain

$$
\begin{gathered}
\dot{\mathcal{R}}(m)=-(k-m+1) r_{2} \mathcal{R}(k)-2 r_{1}\left[\sum_{j=3}^{m} \mathcal{R}(k+j)-\mathcal{R}(k+2) \delta_{m, 1}\left(1-\delta_{m, k}\right)\right]+2 r_{2}[\mathcal{R}(k+m) \\
-\mathcal{R}(k+1)]+\left[(k-m+1) \Delta r-2 r_{1}\right] \mathcal{R}(k+2), \quad \text { for } m \leqslant k \\
\dot{\mathcal{R}}(m)=-\left[(m-k-1) r_{1}+2 r_{2}\right] \mathcal{R}(m) \\
\quad-2 r_{1} \sum_{j=3}^{k-1} \mathcal{R}(m+j)+2 \Delta r \mathcal{R}(k+m), \quad \text { for } m \geqslant k+1
\end{gathered}
$$

where $\Delta r=r_{2}-r_{1}$. These equations can be solved using the same approach used for equations (1) and (2). For $m \geqslant k$ we write

$$
\mathcal{R}\left(m, k, r_{1}, r_{2}, t\right)=\mathrm{e}^{-\left[(m-k-1) r_{1}+2 r_{2}\right] t} G\left(k, r_{1}, r_{2}, t\right), \quad m \geqslant k,
$$

where $G\left(k, r_{1}, r_{2}, t\right)$ is the generalization of $F(k, t)$ in equation (5) with $r_{1}, r_{2}$ :

$$
G\left(k, r_{1}, r_{2}, t\right)=\exp \left[-2 \sum_{j=1}^{k-1} \frac{1-\mathrm{e}^{-j r_{1} t}}{j}+2 \Delta r \frac{1-\mathrm{e}^{-k r_{1} t}}{k r_{1}}\right] .
$$

Substitution of equation (17) into the right-hand side of equation (15) allows us to express the complete solution in integral form analogous to (3) and (4).

\section{Gap distributions in thermally relaxed systems}

Let us now assume that an ensemble of $N k$-mers bound to a chain of $L$ sites is allowed to slide. We can picture an adsorbate with very low diffusivity such that the deposition process can be described as irreversible until saturation is reached, yet such that on much longer time scales particle mobility cannot be ignored and the $k$-mers describe a totally equilibrated system. All configurations consistent with the parameters $\{L, N, k\}$ and open boundary conditions are equally probable, in contrast to the irreversible case.

With a little caution, the gap results we derive in this section can be applied to onedimensional realizations where, instead of sliding, particles are allowed to attach and reattach with rate constants $k_{a}$ and $k_{d}$, respectively. In this scenario, the number of particles is not fixed and a grand canonical description is needed to determine $\langle N\rangle$. For monomers, the Langmuir isotherm gives the equilibrium coverage $L \theta=\langle N\rangle=K_{e q} L\left(1+K_{e q}\right)^{-1}$ with the equilibration constant $K_{e q}=k_{a} / k_{d}$. Since fluctuations scale as $\Delta N /\langle N\rangle=\left(L K_{e q}\right)^{-1 / 2}$, for values of $L K_{\text {eq }}$ large enough, one can assume fluctuations to be small and $\langle N\rangle \sim N$. While these monomer results cannot be trivially extended to $k$-mers, we assume the same trend to hold: namely that for large enough $L K_{e q}$ a system where both attachment and detachment occur can be approximately described by its average number $N$. 
Our goal for the Tonks gas is to evaluate the probability of finding a gap of exact length $m, P_{\text {Tonks }}(m, k)$, given that all configurations are equally probable. This probability distribution function is proportional to the gap density $\rho_{\text {Tonks }}(m, k)$, and will later be compared to the known results from the (finite and infinite) RSA process. We start by evaluating the probability that there are $\ell$ gaps of length $m$, which we define here as $\mathcal{P}(m, \ell, k)$. Consider the partition function for a $\{L, N, k\}$ system:

$$
Z=\sum_{y_{1}=1}^{L-N k+1} \sum_{y_{2}=1+k}^{L-(N-1) k+1} \sum_{y_{3}=y_{2}+k}^{L-(N-2) k+1} \cdots 1=\left(\begin{array}{c}
L-N k+N \\
N
\end{array}\right) .
$$

We now proceed to evaluate the gap distribution $\mathcal{P}(m, \ell)$ in a series of steps. Of the $Z$ configurations, we can select those in which the first through the $\ell$ th gaps are of exactly $m$ sites regardless of the other gaps. This quantity, $\mathcal{Q}(m, \ell, k)$, is determined by fixing the first $\ell$ particles as being adjacent $k+m$-ers and freely distributing the other $N-\ell$ particles along the remainder of the $L$ segment:

$$
\mathcal{Q}(m, \ell, k)=\left(\begin{array}{c}
L-N k-\ell m+N-\ell \\
N-\ell
\end{array}\right) .
$$

This expression is valid up to $N=\ell$ in which case $\mathcal{Q}(m, \ell, k)=1$, as long as $L \geqslant N(k+m)$, otherwise it is 0 . We shall always assume this condition to hold. Note that because of the open boundary conditions, the total number of gaps is $N+1$. For $\ell=N+1$ the above expression is no longer applicable and $\mathcal{Q}(m, N+1, k)=1$ only if $L=N(k+m)+m$, or else it is 0 .

The function $\mathcal{Q}(m, \ell, k)$ fixes the first $\ell$ gaps but does not constrain the remaining $(N-$ $\ell+1)$ which might also be of length $m$. To compute $\mathcal{S}(m, \ell, k)$, the number of realizations of our $\{L, N, k\}$ system with exactly $\ell$ gaps of length $m$, then we must then subtract from equation (20) the configurations with extra $m$-gaps. We obtain

$$
\mathcal{S}(m, \ell, k)=\sum_{\ell_{1}=0}^{N+1-\ell}\left(\begin{array}{c}
N-\ell+1 \\
\ell_{1}
\end{array}\right) \mathcal{Q}\left(m, \ell_{1}+\ell, k\right)(-1)^{\ell_{1}} .
$$

Since we can choose the $\ell$ gaps to be any of the $N+1$ possible ones, the number of configurations where there are only $\ell$ gaps of length $m$ is given by

$$
\mathcal{N}(m, \ell, k)=\left(\begin{array}{c}
N+1 \\
\ell
\end{array}\right) \mathcal{S}(m, \ell, k)
$$

The resulting probability that there are only $\ell_{1}$ gaps of length $m$ is given by normalizing the above with the partition function $Z$ :

$$
\mathcal{P}(m, \ell, k)=\frac{\mathcal{N}(m, \ell, k)}{Z} .
$$

Finally, the probability of having a gap of length $m$ is the average over all possible values of $\ell$ :

$$
P_{\text {Tonks }}(m, k)=\frac{1}{(N+1)} \sum_{\ell=1}^{N+1} \ell \mathcal{P}(m, \ell, k) \text {. }
$$

Our approach yields $P_{\text {Tonks }}(m, k)$ averaged over all possible configurations of the $\{L, N, k\}$ system, even those less likely to occur. In other derivations [21], one constructs the partition function for a subsystem of the original where the number of gaps of length $m$ is fixed at $N_{m, k}$. This is what we calculate as $N_{m, k}=(N+1) P_{\text {Tonks }}(m, k)$. The resulting $Z_{\left\{N_{m, k}\right\}}$ consists of only realizations compatible with this pre-set distribution. The $N_{m, k}$ values that maximize $\ln Z_{\left\{N_{m, k}\right\}}$ are then identified as the equilibrium distribution of the system. Our enumeration procedure differs from this method because it weighs the contribution of all realizations, and 
not just those that carry the highest likelihood. In this sense, although more elaborate, ours is an exact solution that is valid even far away from the thermodynamic limit. What we have illustrated can be generalized to multiple gap lengths to obtain $\mathcal{P}\left(m_{1}, \ell_{1} ; m_{2}, \ell_{2} ; \ldots, k\right)$, the probability for a configuration to have at the same time $\ell_{i}$ gaps of length $m_{i}$ for $i=1,2, \ldots$

The number of gaps of length $m$, per unit length, is obtained by multiplying the gap distribution of equation (24) by the gap density $(N+1) / L$ :

$$
\rho_{\text {Tonks }}(m, k)=\frac{1}{L} \sum_{\ell=1}^{N+1} \ell \mathcal{P}(m, \ell, k) .
$$

It is easy to see that once the form of the partition function $Z$ is determined, the expressions for $P_{\text {Tonks }}(m, k)$ and $\rho_{\text {Tonks }}(m, k)$ follow immediately. Indeed, the building block in determining $P_{\text {Tonks }}(m, k)$ is the function $\mathcal{Q}(m, \ell, k)$, which is simply $Z$ with $L-\ell(k+m)$ substituted for $L$ and $N-\ell$ substituted for $N$. Given the appropriate partition function $Z$, all one has to do, mutatis mutandis, is to repeat the steps leading from equation (20) to equation (23). For example, one can also treat cooperative effects, where two adjacent particles interact with energy $J$. Such configurations carry an additional weight $f=\mathrm{e}^{-J}$ for each interaction. In this scenario, values of $J>0$ tend to cluster the particles, whereas values of $J<0$ will have a spreading effect. The partition function reads

$$
\begin{array}{r}
Z=\sum_{y_{1}=1}^{L-N k+1} \sum_{y_{2}=1+k}^{L-(N-1)} \sum_{y_{3}=y_{2}+k}^{k+1} \ldots\left(1+x \delta_{y_{1}, 1}\right)\left(1+x \delta_{y_{N}, L-k+1}\right) \prod_{j=1}^{N-(N-2) k+1}\left(1+x \delta_{y_{j+1}, y_{j}+k}\right) \\
=x^{N+1} \delta_{L, N k}+(N+1) x^{N}+\sum_{q=0}^{N-1} x^{q}\left(\begin{array}{c}
L-N k+N-q \\
N-q
\end{array}\right)\left(\begin{array}{c}
N+1 \\
q
\end{array}\right)
\end{array}
$$

where $x=f-1$. By setting $x=0$ in this expression one recovers equation (19). This partition function determines the cooperative binding form of $P_{\text {Tonks }}(m, k)$ unambiguously.

In figure 1 , we plot and contrast the exact distribution functions $P_{\text {Tonks }}(m, k)$ and $P_{\mathrm{RSA}}$ $(m, k, \infty)$, as obtained from equations (14) and (24), and the vacancy ratios $m \rho_{\mathrm{RSA}}(m, k, \infty)$ and $m \rho_{\text {Tonks }}(m, k)$, as obtained from equations (12) and (25). To compare the two systems, we choose the same particle length $k=30$, implying a saturated RSA coverage $\theta_{\infty}=0.7548$. At this value of $k$, the Tonks gas corresponding to the RSA process can be characterized by say, $L=596, N=15$. As shown in figure 1, the gap distribution functions are both decreasing as the gap size $m$ increases. Under RSA, gaps exist up to a maximum length $m=k-1$, in contrast to the Tonks gas where more extended vacancies are present as well. The largest RSA gaps occur with a relatively high probability, and contribute heavily to the vacancy ratio $m \rho_{\mathrm{RSA}}(m, k, \infty)$, as can be seen from the inset. Conversely, for the Tonks gas, large gaps carry a much smaller weight and the interplay of large gap size and small probability leads to the non-monotonic behavior of the vacancy ratio, $m \rho_{\text {Tonks }}(m, k)$. Expressions analogous to equations (19)-(25) for particles on a ring are derived in the appendix.

\section{Binding of a second species}

In this section we explore the RSA binding of a second, distinct species on the polymer chain after the adsorption of $k_{1}$-mers. Concurrent deposition of particles of different size has been analysed by other authors [11]. We compare the two distinct limits of irreversibility and equilibration of the first species through, respectively, the saturated RSA gap density $\rho_{\mathrm{RSA}}\left(m, k_{1}, \infty\right)$ and its Tonks gas counterpart, $\rho_{\text {Tonks }}\left(m, k_{1}\right)$. For the latter case, we assume 


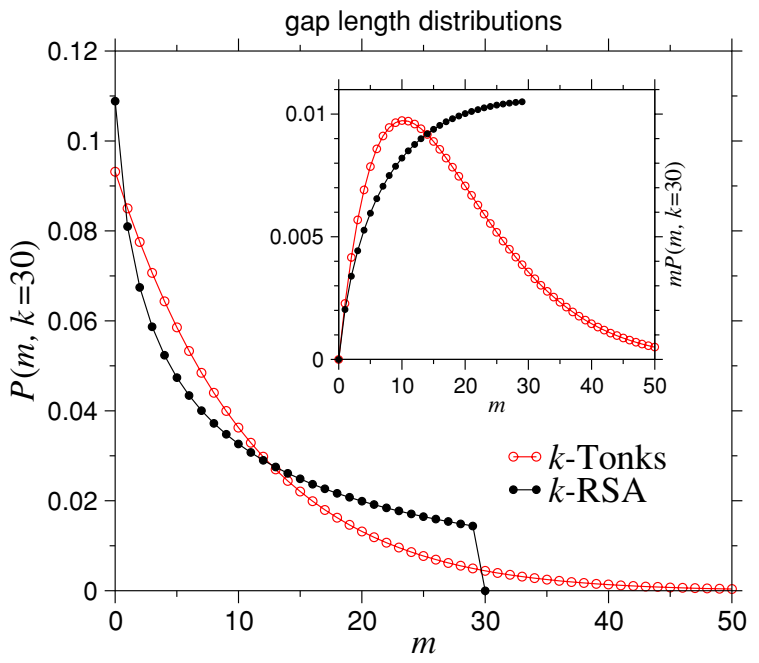

Figure 1. Comparison of the gap distribution functions $\mathcal{P}(m, k)$ for the case of RSA and Tonks gas systems. The impinging particle is of length $k=30$. For the Tonks gas $L=596, N=15$ so that its coverage $\theta=N k / L=0.7550$ matches the corresponding RSA value $\theta_{\infty}(k=30)=0.7548$. The inset shows the vacancy contribution of each $m$ gap, $m \rho(m, k)$ : the area under the two curves gives the vacancy ratio, 0.2452 and 0.2450 for the RSA and Tonks gas, respectively. Note the different trends for RSA and Tonks.

that the equilibrated Tonks gas ensemble is quenched just before deposition of the $k_{2}$-mers. We refer to the irreversible or equilibrated nature of the first deposition process by $k_{1}-\mathrm{RSA}$ and $k_{1}$-Tonks, respectively.

The segments onto which the second species impinge are the finite-sized gaps left by the first deposition process. For $k_{2}$-mers we need to consider the finite-size description illustrated by equations (6)-(8). The question we intend to address is how does the secondary deposition process on the $m$-gaps left by the first species increase the coverage in the two distinct cases of irreversible RSA and Tonks gas gap distributions. Under RSA of $k_{2}$-mers, the fraction of empty sites on a finite-sized gap of length $m$ is given by equation (9): $\mathcal{E}\left(m, k_{2}, t\right)=\sum_{j=1}^{m} j \rho_{m}\left(j, k_{2}, t\right)$. The time-dependent coverage of the entire chain is the average over all such gaps of length $m$ weighted by the gap density. For the RSA-saturated $k_{1}$ deposition process we have

$$
\theta_{\mathrm{RSA}}\left(k_{1}, k_{2}, t\right)=1-\sum_{m=1}^{k_{1}-1} \mathcal{E}\left(m, k_{2}, t\right) \rho_{\mathrm{RSA}}\left(m, k_{1}, \infty\right) .
$$

Conversely, for the Tonks gas,

$$
\theta_{\text {Tonks }}\left(k_{1}, k_{2}, t\right)=1-\sum_{m=1}^{L-N k} \mathcal{E}\left(m, k_{2}, t\right) \rho_{\text {Tonks }}\left(m, k_{1}\right) .
$$

From both these equations we can obtain the reaction rate $\xi\left(k_{1}, k_{2}, t\right)=\dot{\theta}\left(k_{1}, k_{2}, t\right)$. In figure 2 we plot total coverage as a function of time for both $k_{1}$-RSA and $k_{1}$-Tonks gap distributions as given by equations (27) and (28). We set $k_{1}=30$, and select a few representative values of $k_{2}$. The Tonks gas is chosen so that its initial coverage corresponds (up to a few parts per thousand) to the same coverage as the RSA system. A few features are worth noticing: the initial rate, the derivative of the coverage at $t=0$, is a non-monotonic function of particle 


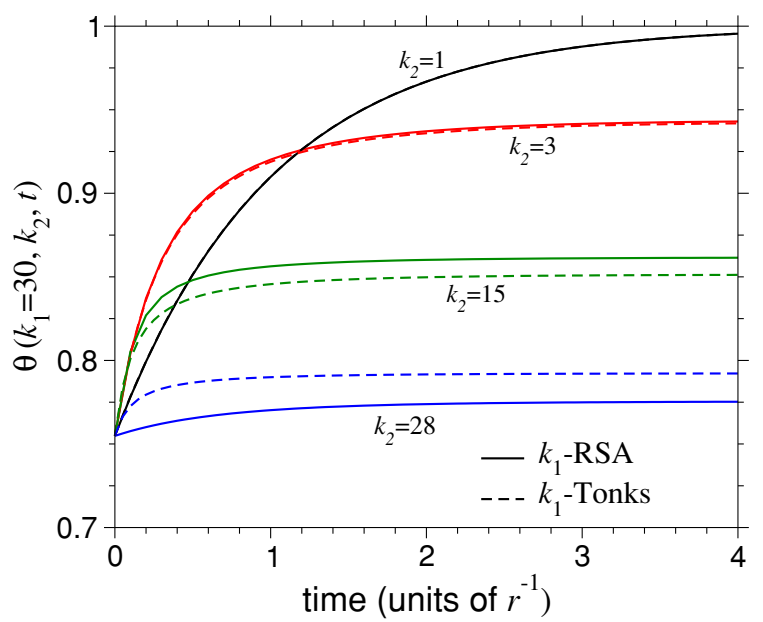

Figure 2. Total coverage as a function of time due to deposition of a second $k_{2}$ particle. The initial gap distributions are generated by saturated-RSA of $k_{1}=30$ or by a Tonks gas of equivalent coverage. We choose the Tonks gas with $L=596, N=15, k_{1}=30$ so that its coverage $\theta=N k_{1} / L=0.7550$ matches the corresponding saturated RSA case $\theta_{\infty}\left(k_{1}=30\right)=0.7548$. Solid and dashed curves correspond to $k_{1}$-RSA and $k_{1}$-Tonks initial gap distributions, respectively, The value of $k_{2}$ decreases from bottom to top : $k_{2}=28,15,3$, for each pair of curves from the bottom up. For $k_{2}=1$, the two curves (Tonks and RSA) lead to perfect filling, saturating at 1 and are indistinguishable. Note the non-monotonic initial rate as a function of $k_{2}$.

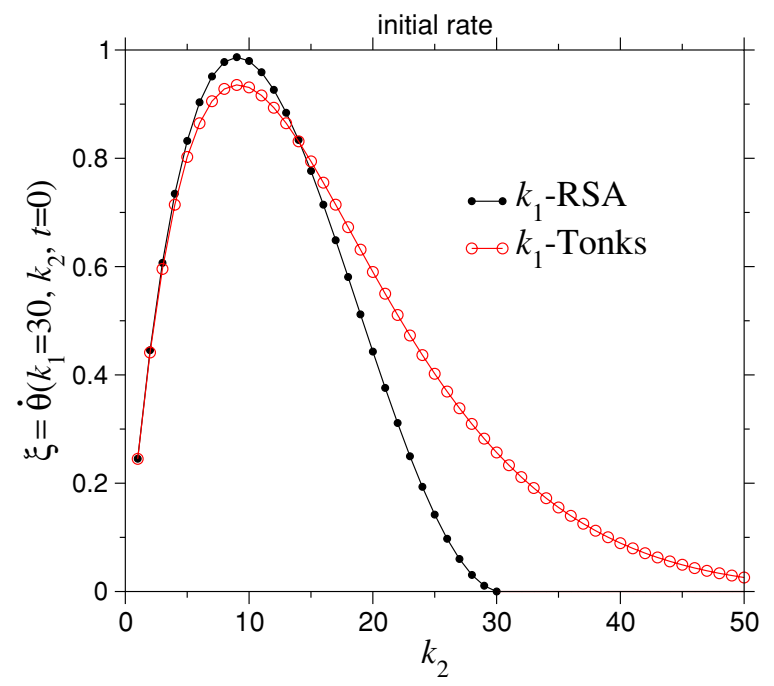

Figure 3. Reaction rate at $t=0$ as a function of $k_{2}$ and for the initial deposition of $k_{1}=30$ both for the $k_{1}$-RSA and $k_{1}$-Tonks generated initial distributions.

size $k_{2}$; the final coverage is greater for RSA than for Tonks systems, unless the size of the $k_{2}$-mer is not too dissimilar from $k_{1}$, or $k_{2} \geqslant k_{1}$. These are all consequences of the gap distributions of figure 1 . Let us focus on them in more detail. In figure 3 we plot the initial rate $\xi=\dot{\theta}\left(k_{1}, k_{2}, t=0\right)$ as a function of $k_{2}$; the initial reaction rate is indeed non-monotonic as a function of $k_{2}$. Both very small and very large values of $k_{2}$ signify little growth activity, 


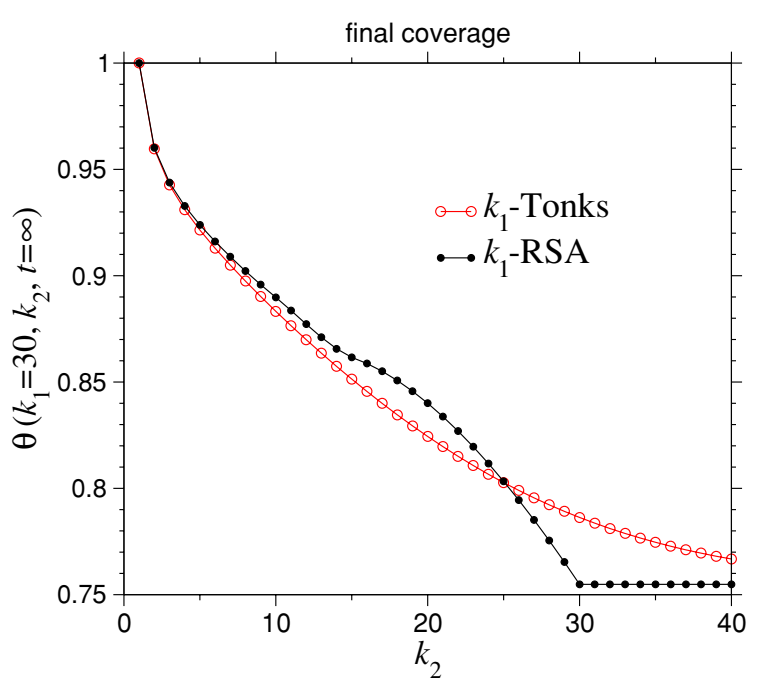

Figure 4. Saturation after deposition of the second particle species as a function of $k_{2}$, and for the initial deposition of $k_{1}=30$ both for the $k_{1}$-RSA and $k_{1}$-Tonks generated initial distributions. The Tonks gas parameters are $\{L=596, N=15\}$.

in the first case because the contribution to the coverage from short segments is small, and in the second case because large $k_{2}$ values are difficult to accommodate.

In figure 4 we plot the coverage due to adsorption of the second species, and show that major differences arise when the size of $k_{2}$ is similar to, or exceeds $k_{1}$. These trends are a direct consequence of the different initial $k_{1}$ gap distributions plotted in figure 1 . For values of $k_{2} \geqslant k_{1}$, the $k_{1}$-Tonks gap distribution is the only one that can allow binding of $k_{2}$-mers, and in this case, the total coverage is higher than for $k_{1}$-RSA. When $k_{2} \lesssim k_{1}$ however, the $k_{1}$-RSA system allows for a much higher fraction of gaps on which to deposit $k_{2}$-mers compared to $k_{1}$-Tonks. A crossover arises when the higher $k_{1}$-RSA probability for smaller gaps compensates the long tail in the $k_{2}$-Tonks gap distribution function. For values of $k_{2} \ll k_{1}$, the $k_{2}$-mer is too small to allow for substantial distinctions between the two gap distributions.

\section{Summary and conclusions}

In this paper we have studied a one-dimensional lattice adsorption process in the two limits of totally irreversible and fully equilibrated deposition. For the equilibrated Tonks gas case, we calculated the exact gap distribution function $P_{\text {Tonks }}(m, k)$ with and without cooperative effects, valid for any choice of the parameters $\{L, N, k\}$, and in the case of open and periodic boundary conditions. The gap distribution function computed in equation (23) is intimately connected to the cluster distribution defined as the probability of having $n_{c}$ clusters made of contiguous $k$-mers. Indeed, an $n_{c}$ cluster configuration corresponds to one with $N-n_{c}$ gaps of zero length. The cluster distribution function is simply $\mathcal{P}\left(0, N-n_{c}, k\right)$ of equation (23).

The gap distribution function of the Tonks gas is compared to that known from RSA processes with identical coverages, $N k=\theta_{\infty}(k) L$. We have shown that strong differences exist between the two cases, and that such differences are reflected in the adsorption of a second species onto the remaining gaps. Final coverages, reaction rates and allowed values of $k_{2}$ are all tests that could be exploited to determine the particle mobility of the first species. 
Cooperative effects for both the initial RSA and Tonks-gas systems can be incorporated as well, in the first case by introducing the $r_{1}, r_{2}$ rates of adsorption on an open or a neighbouring edge, and in the second by considering an extra Boltzmann weight $f$ every time two particles touch.

In this work we have chosen $k_{1}=30$ and $k_{2}=3$ to represent the size of the SSB and Rec-A proteins non-specifically adsorbing on DNA. Our results can be used as a guide to determine, by analysing the subsequent deposition of Rec-A, whether or not SSB deposition is irreversible. The dependence of Rec-A coverage as a function of Rec-A-SSB cooperativity can also be found. Additional biological complexities, however, pose certain limitations on this study. For example, Rec-A is able to displace SSB upon contact, a feature we have neglected. Nonetheless, our work can be considered as a first step in analysing interactions between SSB and Rec-A particles. We plan to explore such 'knock-out' phenomena in future work. The results we have presented may also be useful in determining the Langmuir isotherm for discrete lattice systems where the adsorbing species has length $k>1$.

\section{Acknowledgments}

MRD acknowledges support from the Laboratory for Molecular Sciences at Caltech and the US National Science Foundation through grant NSF CHE-0117850. TC acknowledges support from the NSF through grants DMS-0206733 and DMS-0349195 and from the National Institutes of Health through grant K25AI058672-01.

\section{Appendix. Periodic boundary conditions}

When dealing with lattices with periodic boundary conditions, or rings, the equations must be modified to include end effects. The partition function for a Tonks gas on a ring reads

$$
Z_{\text {ring }}=\frac{L}{N}\left(\begin{array}{c}
L-N k+N-1 \\
N-1
\end{array}\right) \text {. }
$$

The factor $L / N$ arises because the first $k$-mer particle can be deposited anywhere on the $L$ ring and that there are $N$ rods to choose from. The second term is the open boundary condition contribution for the remaining $N-1$ particles on a segment of length $L-k$. To find the number of configurations of exactly $\ell$ gaps of length $m$ on a ring, let us envision a pseudo-particle made of two $k$-mers separated by a gap of length $m$. This particle is of length $2 k+m$ and placing it anywhere on the ring leaves us with an open boundary chain of length $L^{\prime}=L-(2 k+m)$ on which to place $N^{\prime}=N-2$ particles and $\ell-1$ gaps of length $m$. Let us also specify the $L, N$ dependence in both $\mathcal{N}_{\text {ring }}$ and the open boundary $\mathcal{N}$ of equation (22):

$$
\mathcal{N}_{\text {ring }}(m, \ell, L, N, k)=\frac{L}{\ell} \mathcal{N}\left(m, \ell-1, L^{\prime}, N^{\prime}, k\right)
$$

This expression is valid for $\ell>1$. For $\ell=1$, after placing the pseudo-particle, we ask for the number of configurations on an open boundary system where of all gaps present, none are of length $m$. This is the line partition function of equation (19) with all contributions from $m$-gaps eliminated. We call the latter function $T(m, L, N, k)$ :

$$
T(m, L, N, k)=\sum_{\ell=1}^{N+1}\left(\begin{array}{c}
N+1 \\
\ell
\end{array}\right) R(m, \ell, k)
$$

where $R(m, \ell, k)$ is given from equation (21). Hence,

$$
\mathcal{N}_{\text {ring }}(m, 1, L, N, k)=L\left[\left(\begin{array}{c}
L^{\prime}-N^{\prime} k+N^{\prime} \\
N^{\prime}
\end{array}\right)-T\left(m, L^{\prime}, N^{\prime}, k\right)\right] .
$$


Finally we obtain the probability of finding a gap of exactly length $m$ on an $L$ ring as

$$
\mathcal{P}_{\text {ring }}(m, \ell, L, N, k)=\frac{1}{N Z_{\text {ring }}} \sum_{\ell=1}^{N} \ell \mathcal{N}(m, \ell, L, N, k) .
$$

This quantity should be seen as the equivalent of equation (24) for the open boundary system. The gap density is now $N / L$.

\section{References}

[1] Bartelt M C and Privman V 1991 Int. J. Mod. Phys. B 52883

[2] Evans J W 1993 Rev. Mod. Phys. 651281

[3] Talbot J, Tarjus G, Van Tassel P R and Viot P 2000 Colloids Surfaces A 165287

[4] Schaaf P, Talbot J, Raebony H M and Reiss H 1988 J. Phys. Chem. 924826

[5] Flory P J 1939 J. Chem. Phys. 611518

[6] Cohen E R and Reiss H 1963 J. Chem. Phys. 38680

[7] Renyi A 1958 Pub. Math. Inst. Hung. Acad. Sci. 3109

[8] Onoda G Y and Liniger E G 1896 Phys. Rev. A 33715

[9] Widom B 1966 J. Chem. Phys. 443888

[10] Gonzales J J, Hemmer P C and Hoye J S 1974 Chem. Phys. 3228

[11] Bartelt M C and Privman V 1991 Phys. Rev. A 44 R2227

[12] Tarjus G, Schaaf P and Talbot J 1990 J. Chem. Phys. 938352

[13] Ramsden J J 1993 Phys. Rev. Lett. 71295

[14] Feder J and Giaver I 1980 J. Colloid Interface Sci. 78144

[15] von Hippel P H and Berg O G 1989 J. Biol. Chem. 264675

[16] Thompson C 1988 Classical Statistical Mechanics (Oxford: Clarendon Press)

[17] Bonnier B, Boyer D and Viot P 1994 J. Phys. A: Math. Gen. 273671

[18] Meeron E A and Siegert A J F 1968 J. Phys. Chem. 483139

[19] Kowalczykowski S C and Krupp R A 1987 J. Mol. Biol. 19397

[20] Kowalczykowski S C, Clow J, Somani R and Varghese A 1987 J. Mol. Biol. 19381

[21] Soto-Campos G, Bowles R, Itkin A and Reiss H 1999 J. Stat. Phys. 96111

[22] Boucher E A 1978 Prog. Poly. Sci. 663

[23] Boucher E A 1972 J. Chem. Soc. Faraday Trans. 721697

[24] Boucher E A 1973 J. Chem. Phys. 593848

[25] Bartelt M C 1991 Phys Rev A 433149 